\title{
Prise en charge des troubles de la reproduction chez l'adolescent
}

\author{
N. FRANÇOIS 1,2, J.M. KUNSTMANN2
}

1 Rééducation Fonctionnelle, CMPA, Neufmoutiers-en-Brie - 2CECOS Hôpital Cochin, Paris.

\section{RÉSUMÉ}

L'adolescence est une période de recherche d'identité sexuée. Comment se construire sur ce plan lorsqu'on est un jeune para ou tétraplégique? Comment les soignants peuvent-ils l'aider à résoudre ce questionnement et à quel stade de l'évolution de son handicap?

Nous abordons dans un premier temps la question de l'érection et de la qualité des rapports et dans un deuxième temps celle de la procréation.

II s'agit principalement d'une information concrète, suffisamment tôt, adaptée à la définition neurologique de la para ou tétraplégie et surtout d'une action de conservation préventive de sperme avant que ne surviennent les complications infectieuses et urologiques. Nous faisons part de notre expérience auprès de 24 adolescents blessés médullaires lors de nos consultations spécialisées au CMPA de Neufmoutiers et au CECOS de I'hôpital COCHIN de Paris.

Mots-clés : Procréation, Prévention, Adolescent, Blessés médullaires.

\section{INTRODUCTION}

Rappelez-vous ! Nous sommes tous passés par ce stade de l'adolescence et beaucoup ont connu ou connaissent en tant que parents ces difficultés relationnelles auprès d'enfants devenus grands qui cherchent leur identité.

L'adolescence est un passage. L'adolescent n'est plus un enfant, il n'est pas encore un adulte. Souvent il se pose la question : « suis-je normal ?».
L'adolescence est une période où la tolérance à la maladie décroît. La paraplégie est une injustice supplémentaire pouvant engendrer un rejet de la maladie ou du traitement. La relation de l'adolescent à son corps n'est jamais facile. Les craintes d'un impact du handicap médullaire sur les fonctions sexuelles et la capacité de reproduction sont très souvent présentes chez eux.[1]

Toutefois, devant une maladie qui va devenir chronique, vis à vis de la sexualité certains adolescents ne demandent rien à priori. La plupart n'a pas encore eu de rapports sexuels. Une image négative du corps perturbe la sexualisation et peut entraîner un évitement farouche de la sexualité, l'adolescent se sentant incapable de séduire ou d'être désiré. Parfois, l'évitement de la sexualité fait écho à un souhait des parents, voire à une tendance des soignants pour lesquels il est plus simple de continuer à traiter un enfant malade qu'un adolescent avec lequel il faut négocier de personne à personne et parfois difficilement.

Traversant son adolescence sans la vivre, il fait peut être l'économie immédiate d'une crise mais cette économie risque de s'avérer désastreuse à long terme pour l'adulte qu'il est en passe de devenir.

Pour d'autres, le handicap neurologique fait naître un certain nombre d'inquiétudes au sujet de la qualité de la vie sexuelle et de la fertilité ou même de la normalité de l'enfant à naître.

Correspondance :

N. François. Centre Médical et Pédagogique pour Adolescents 77610 Neufmoutiers-en-Brie

Tél. 0164424646 ; Fax. 0164424604 ; e-mail :

fsef.cmpa@wanadoo.fr

Communication à la Session SALF-AFU des $6^{\circ}$ Journées de la Fédération Française d'Étude de la Reproduction, Lyon, 5-7 septembre 2001 
Il importe donc de savoir anticiper ces problèmes et de fournir aux jeunes blessés médullaires les informations les plus claires et les plus précises sur leur état et d'en préciser le retentissement éventuel sur leur fonction sexuelle.

\section{QUI CONSULTE?}

Dans le cadre de la consultation ouverte au CECOS de COCHIN pour la prise en charge des blessés médullaires, 24 adolescents para ou tétraplégiques ont consulté.

La moyenne d'âge au moment de l'accident s'est située à 16 ans avec des extrêmes de 2 ans à 21 ans.

La définition neurologique était la suivante :

17 tétraplégiques dont 13 complets et 4 incomplets

4 paraplégiques au-dessus de D10 complet

1 entre D11 et L2 incomplet

2 syndromes de la Queue de Cheval incomplets

\section{La paraplégie est survenue :}

dans 14/24 cas lors d'un accident de la voie publique dont 9 accidents de voiture

5 accidents de deux roues

4 plongeons

4 chutes

2 maladies (rupture vasculaire)

Les déplacements du para ou tétraplégique s'effectuaient:

pour 18 en fauteuil roulant manuel,

3 en fauteuil roulant électrique,

et pour 3 en marchant avec des appareils.

\section{Sur le plan urologique :}

3 sont autonomes pour les mictions, et ne portaient pas de collecteur et n'avaient que très rarement des fuites urinaires à l'effort important.

11 pratiquaient des auto-sondages, 10 des hétéro-sondages.

\section{LE CONTENU DES CONSULTATIONS SPÉCIALISÉES}

Le délai entre l'apparition de la paraplégie et la première consultation concernant la fonction génito-sexuelle était en moyenne de 4 ans avec des extrêmes de 5 mois après l'accident à 16 ans après l'accident.

Ces grands écarts s'expliquent d'une part par les différences d'âge à la survenue de l'accident (pour deux au moment de la petite enfance à 2 et 3 ans d'où pour eux le grand délai avant la première consultation sur le sujet) pour d'autres la préoccupation survient tôt après l'accident : « Docteur est ce que je remarcherai, et est ce que çà remarchera ? »

\section{Concernant la Sexualité :}

Après un entretien consacré essentiellement à l'information une évaluation neurologique a été faite pour vérifier le niveau métamérique, la hauteur du syndrome lésionnel flasque et l'étendue du syndrome sous lésionnel spastique.[3].

Pour 10 patients l'entretien s'est limité à une information sur la sexualité et la procréation.

Pour les 14 autres, ceux qui étaient motivés et volontaires, des essais de stimulation de l'éjaculation ont été entrepris avec toutes les précautions psychologiques nécessaires.

$\mathrm{Au}$ moment de la consultation, 11/24 vivaient chez leurs parents et $8 / 24$ en institution, 5/24 étaient autonomes dans un appartement avec une tierce personne.

4/24 avaient une vie de couple stable

$8 / 24$ avaient eu des rapports depuis l'accident.

Le mode d'obtention de l'érection est psychogène pour 3 , réflexe pour 20 ; mais il faut signaler que pour 6 jeunes la question du lien entre l'érection et les rapports sexuels semblait prématurée.

Le recours aux facilitateurs de la qualité de l'érection sont classiques : Soit par VIAGRA de préférence à $50 \mathrm{mg}$, soit par injections inra-caverneuses de Caverject à $10 \mathrm{vg}$, soit par vibromassage à l'aide du Ferticare Personnal.

\section{Concernant la Procréation :}

Il s'agit avant tout, grâce à une information adaptée à chaque cas, de faire comprendre à chacun l'intérêt d'une conservation spermatique de précaution avant que ne surviennent les complications infectieuses urologiques (infections urinaires récidivantes, prostatites sur sondages pluriquotidiens, parfois orchites récidivantes)[2]. Dans notre série, 1 seul couple avait un projet parental à moyenne échéance.

Nous utilisons les moyens de stimulations mécaniques classiques chez le paraplégique avec au préalable déclenchement de l'érection par stimulation locale aidée ou non de Viagra ou de Caverject aux doses décrites ci dessus. Il faut signaler que nous n'avons jamais, pour ces adolescents, utilisé l'électrostimulation intra rectale comme mode de stimulation.

Sur les 14 patients, du sperme a pu être recueilli dans 10 cas.

Les caractéristiques du sperme recueilli chez ces adolescents blessés médullaires sont les suivantes données dans le Tableau 1.

Ces résultats sont très comparables à ceux observés chez l'adulte para ou tétraplégique en fonction des modes de stimulation utilisés. 
Nous n'avons pas pratiqué de façon systématique des dosages de FSH, $\mathrm{LH}$, et testosterone circulantes ou la recherche des anticorps antispermatozoïdes, mais les trois fois où cela a été fait chez des adolescents dont l'accident était survenu dans la petite enfance les taux à 16 ou 18 ans étaient normaux.

\section{POUR CE QUI EST DE L'ANALYSE DES ÉCHECS : (4 CAS / 14).}

2 patients avaient un niveau neurologique D12/L2. ce qui rend les stimulations mécaniques aléatoires.

Pour 1 patient, on a obtenu une seule fois quelques rares spermatozoïdes immobiles mais le jeune n'a pas donné suite.

Dans un cas, la stimulation a été rendue difficile par la présence d'une pompe à Liorésal implantée non modifiable dans son débit ; ainsi l'arc réflexe peut être déclenché mais la moelle ne réagit pas de façon suffisamment intense du fait de l'imprégnation du Liorésal à un taux efficace pour les contractures mais gênant pour la stimulation.

\section{DEUX CAS PARTICULIERS}

Il s'agit d'un jeune tétraplégique et d'un jeune paraplégique haut D2 qui ont été victimes, l'un et l'autre, d'un accident de la voie publique alors qu'ils étaient âgés de 2 et 3 ans. Ce n'est qu'à l'âge de 17 ans pour l'un et pour l'autre que le problème génito-sexuel a été abordé :

- Le paraplégique utilise du VIAGRA et fait l'expérience de rapports et a pu mettre en banque 14 paillettes de sperme de façon préventive n'ayant pas de projet parental immédiat. Ses dosages hormonaux sont normaux en ce qui concerne FSH, LH et testostérone.

Le jeune tétraplégique qui est encore en institution n'a pas de projet particulier sur le plan génito-sexuel et n'a pas voulu encore aborder le problème de la qualité des rapports et la mise en banque. Il faut dire que le niveau métamérique est à $\mathrm{C} 5$ et que toute stimulation peut déclencher une manifestation d'hyperréflectivité autonome un peu difficile à contrôler. Pour l'instant, FSH, LH et testostérone sont normales.

\section{CONCLUSION}

Pour nous, il est nécessaire de pratiquer une information et des stimulations de l'éjaculation à visée préventive avant que ne surviennent les complications urologiques infectieuses classiques : infections urinaires, prostatiques et parfois survenues d'orchite comme cela se retrouve dans 1/4 des cas lors de sondages pluri-quotidiens au long cours. Cette prévention utile n'est souvent pas en phase avec la maturation psychologique de l'adolescent pourtant nécessaire à un passage à l'acte médical. De plus, sur le plan pratique, il est bon d'apprendre aux jeunes patients de ne pas tenir ses testicules coincés au chaud entre ses cuisses sur un coussin en plastique de fauteuil roulant.

L'approche du retentissement génito-sexuel de l'état de para ou tétraplégie est particulièrement délicate chez l'adolescent dans la mesure où les difficultés liées à l'adolescence se présentent à un moment où le handicap arrive en même temps que s'élabore un travail de construction d'une personne sexuée et ce, de façon habituellement chaotique : «qui suis-je ? » « Je veux et je ne veux pas... ». Il faut donc laisser un certain temps au travail de l'information et de la réflexion pour faciliter la prise en compte de tous les paramètres du retentissement de la lésion médullaire sur la nouvelle physiologie et faire en sorte qu'il se dirige vers une « personne ressource » connue du service dans ce domaine.

Cet adolescent qui a perdu sa sensibilité au niveau des organes génitaux et qui souvent ne peut pas se souvenir d'un vécu dans ce domaine, fait appel à la consultation spécialisée pour donner « du sens » à son questionnement sur une future vie de couple, la qualité des rapports ou les possibilités de procréation [4].

Ainsi, la collaboration étroite des services de rééducation à orientation neurologique avec le CECOS à l'intérieur de ses locaux, grâce à une unité de lieu et une unité d'action, nous semble une des clés de la qualité de cette prise en compte du retentissement génito-sexuel de ces jeunes blessés médullaires. 24 patients ont reçu une information, 14 étaient intéressés par une conservation spermatique, 10 ont pu obtenir un recueil avec conservation spermatique de précaution.

L'approche rééducative du retentissement génito-sexuel chez l'adolescent au cours de son premier séjour en centre de rééducation fait partie de son apprentissage d'homme para ou tétraplégique grâce à des actions pédagogiques de prévention qu'il doit assimiler pour qu'avant sa sortie définitive de l'établissement il puisse avoir suffisamment d'acquis pour construire sa nouvelle personnalité, connaître sa nouvelle physiologie et ainsi bâtir des projets et s'intégrer dans la société.

\section{RÉFÉRENCES}

1. ATHEA N., ALVIN P., COURTECUISSE V. : sexualisation et sexualité chez les adolescents porteurs de maladie chronique. Médecine et Hygiène. 14 novembre 1990. Genève

2. FRANCOIS N. : troubles génito-sexuels et procréation des blessés médullaires. Revue du Praticien, 1995, 2017-2021

3. JOUANNET P. ; CHAPELLE A. ; FRANCOIS N. ; HEILFORN. ; ROUFFET A.J.. La fonction génito-sexuelle du paraplégique, La Paraplégie Marc Maury,Flammarion Médecine Sciences : 404-420.

4. SOULIER B. Aimer au-delà du handicap.Vie affective et sexualité du paraplégique. Pratiques sociales - Privat. 


\begin{abstract}
Alteration of reproductive function in spinal cord injured teenager
\end{abstract}

N. FRANCOIS, J.M. KUNSTMANN

Adolescence is a period marked by the search for sexual identity. How do paraplegic or quadriplegic teenagers develop their sexual identity? How can the nursing team help them in this process?

The authors study erection and the quality of sexual relations and then examine the problem of procreation.

Early, practical information adapted to the neurological definition of the spinal cord injury is essential, with preservation of sperm prior to the development of urological and infectious complications.

The authors report their experience of 24 paraplegic or tetraplegic teenagers at the CMPA in Neufmoutiers and the CECOS at Cochin Hospital in Paris.

Key words : Procreation, Prevention, Teenagers, Spinal cord injury

Tableau 1. Caractéristiques spermatiques des 10 cas avec prélèvements positifs. Il s'agit de valeurs moyennes lorsque le patient a fait plusieurs prélèvements et recueils. NB : Les quatre chiffres de la mobilité correspondent de gauche à droite et de haut en bas à a) Mobilité très rapide, b) Mobilité moyenne, c) Mobilité sans déplacement, d) Absence de mobilité, suivant la classification de l'OMS.

\begin{tabular}{|c|c|c|c|c|c|c|c|c|c|c|c|}
\hline $\begin{array}{c}\text { Nombre de } \\
\text { prélèvements }\end{array}$ & 8 & 3 & 3 & 6 & 1 & 1 & 1 & 1 & 2 & 4 & 3 \\
\hline $\begin{array}{c}\text { Volume en } \\
\text { ml }\end{array}$ & 1,30 & 0,35 & 0,40 & 1,70 & 1,70 & 0,50 & 0,80 & 2,70 & 1,80 & 2,50 & 1,38 \\
\hline Numération & 50 & 413 & 150 & 100 & 213 & 100 & 72 & 110 & 300 & 520 & 202,8 \\
\hline $\begin{array}{c}\text { Mobilité } \\
\text { initiale }\end{array}$ & $\begin{array}{c}20 / 2 / \\
0 / 78\end{array}$ & $\begin{array}{c}0 / 20 / \\
5 / 75\end{array}$ & $\begin{array}{c}10 / 3 / \\
2 / 85\end{array}$ & $\begin{array}{c}5 / 15 / \\
7 / 75\end{array}$ & $\begin{array}{c}10 / 20 / \\
5 / 65\end{array}$ & $\begin{array}{c}2 / 8 / \\
5 / 85\end{array}$ & $\begin{array}{c}10 / 35 / \\
2 / 52\end{array}$ & $\begin{array}{c}1 / 1 / \\
1 / 97\end{array}$ & $\begin{array}{c}0 / 7 / \\
3 / 90\end{array}$ & $\begin{array}{c}20 / 7 / \\
3 / 70\end{array}$ & \\
\hline $\begin{array}{c}\text { Nombre de } \\
\text { doses } \\
\text { conservées }\end{array}$ & 40 & 27 & 17 & 14 & 20 & 3 & 8 & 33 & 12 & 38 & 21 \\
\hline
\end{tabular}

